\title{
Application of Open-air Capacitive Sensors for Voltage Monitoring near Terminations in HV and EHV Insulated Connections
}

\author{
P.A.A.F. Wouters ${ }^{1}$, F. Barakou ${ }^{1}$, E.F. Steennis ${ }^{1,2}$ \\ ${ }^{1}$ Eindhoven University of Technology, Eindhoven, the Netherlands \\ ${ }^{2}$ DNV GL, Arnhem, the Netherlands
}

\begin{abstract}
Contactless capacitive sensors are applied to monitor (over)voltages near the overhead line terminations of a substation or at cable to line transitions. The sensor response is the signal time derivative when loaded with a resistive impedance and the waveform is restored by integration. As part of this differentiating/integrating $(\mathrm{D} / \mathrm{I})$ measuring concept, the use of open-air sensors results in excellent EMC characteristics but the inherent cross-coupling to other phases has to be dealt with. Three applications are presented: 1) For a $150 \mathrm{kV}$ cable termination the partial discharge activity needs to be related to momentary phase voltages; 2) Measured slow front overvoltage at a $380 \mathrm{kV}$ cable termination from line energization are compared with predictions from numerical simulation; 3 ) The perspectives of employing the D/I method for (very) fast front overvoltages near a $380 \mathrm{kV}$ circuit breaker are examined.
\end{abstract}

\section{Introduction}

Terminations at the end of a transmission line or at a transition between overhead line and underground power cable are critical components in electrical insulation. Monitoring of the high voltage (HV) and extra high voltage (EHV) terminations may serve different purposes. It can provide a reference base e.g. when performing partial discharge (PD) diagnostics. For PD interpretation, one needs to distinguish internal discharges arising inside the terminations from corona discharges coming from the connecting overhead lines. To this end, the PD activity can be correlated to the momentary angle of the phase voltages. Voltage monitoring can also provide information on the exposure of the insulation to slow or fast front transient overvoltages. Such overvoltage types arise e.g. from line energization and can cover a wide frequency spectrum ranging from power frequency to $100 \mathrm{MHz}[1]$.

The installation of equipment such as RC-dividers capable to capture high-frequency signals is costly, leaves a relatively big footprint and should be planned ahead. After an operation time, e.g. to verify the absence of PD activity or to confirm that overvoltages remain within safe limits, there might be no further need for having such dedicated equipment installed. Dividers can also be prone to electromagnetic compatibility (EMC) disturbance [2], e.g. arising from large ground currents upon a switching event. A non-invasive, cost-efficient, easy to (re-) install, EMC-proof measurement system would therefore be appealing to occasionally perform voltage monitoring.
This paper describes applications based on open-air capacitive sensors as part of a differentiating/integrating (D/I) measurement principle. One of the earlier papers on the method is provided in [3]. As the sensors basically consist of a metal plate which forms a capacitance to high-voltage conductors in the vicinity, the requirements related to costs, installation effort and being non-invasive are fulfilled. A drawback is that openair sensors are not selective as they have capacitive coupling to any phase conductor in the vicinity. A matrix containing all couplings between sensors and phases needs to be established to entangle the cross-coupling.

A number of open-air sensor applications with solutions for the selectivity problem can be found in literature. In [4] line energization is studied. Decoupling was achieved based on the fact that different phases had different contact moments. This allowed to determine the responses of the sensors near each phase upon the three distinct energization moments. From this information the coupling matrix was established. In [5] the capacitive sensors were inside a GIS system and in prior tests the phases were energized separately. A harmonics study was published in [6] where sensors were placed halfway between two overhead line pylons. The capacitances were derived from the equations for cylindrical line conductors above perfect conductive earth. Unknowns in distances (e.g. line heights) could be resolved by assuming symmetric power frequency voltages on the overhead lines and fitting the measured and calculated waveforms. For the transient overvoltage study in [7] a similar approach was taken. However, the sensors were placed near the terminations for high sensitivity. Due to the complex termination design, analytical field calculation was not possible, leaving the coupling matrix underdetermined. It was shown that with a few assumptions, allowing for large uncertainty margins, the waveforms could be restored with satisfactory accuracy $[7,8]$. A similar approach is adopted here.

\section{EMC aspects of the $D / I$ methodology}

The $\mathrm{D} / \mathrm{I}$ configuration with a single capacitive electrode is shown in Figure 1a. The electric field from the HV conductor induces a current in the capacitive sensor. Its magnitude is determined by the capacitance $C_{H V}$ between the $\mathrm{HV}$ conductor and the sensor and causes a voltage over the resistor $R_{m}$ at the end of the measurement cable. The capacitance value depends on distances and layout, and also on conducting objects in the neighbourhood. Typically, the sensor area can be chosen such that the capacitance is in the range of about 0.1 to $10 \mathrm{pF}[2,7]$ 
for (E)HV systems. The sensor plate also has a capacitance to earth, $C_{E}$ in parallel to $R_{m}$, but as its value is low it usually has negligible effect.

In comparison with capacitive dividers the $\mathrm{D} / \mathrm{I}$ principle differs in the following EMC aspects:

- As shown with (1) up to a cut-off frequency, which depends on design parameters, the signal response over the measurement resistance $R_{m}$ is the time derivative. High frequency noise entering the measurement cable will add to the sensor signal, but its effect will be minimized due to the subsequent integration step.

- Large ground currents may arise as a consequence of e.g. line energization. A common mode $(\mathrm{CM})$ current $I_{C M}$ is induced in ground structures partly flowing along the measurement cables, Figure 1b. Depending on the cable quality, expressed as its transfer impedance $Z_{t}$, a differential mode voltage $U_{D M}$ arises, (2), which adds to the sensor signal [9]. The impedance at the receiving end is chosen to be small compared to the impedance $Z_{E}=1 / \omega C_{E}$ at the sending end of the measurement cable for frequencies of interest. The differential mode voltage arising from the ground current divides over these end impedances. The major fraction drops over the highest impedance which is located at the sensor side, whereas at the measurement side only a small fraction arises. This is different from a capacitive divider where, in order not to load the low-voltage branch of the divider, the receiving end impedance is taken relatively large.

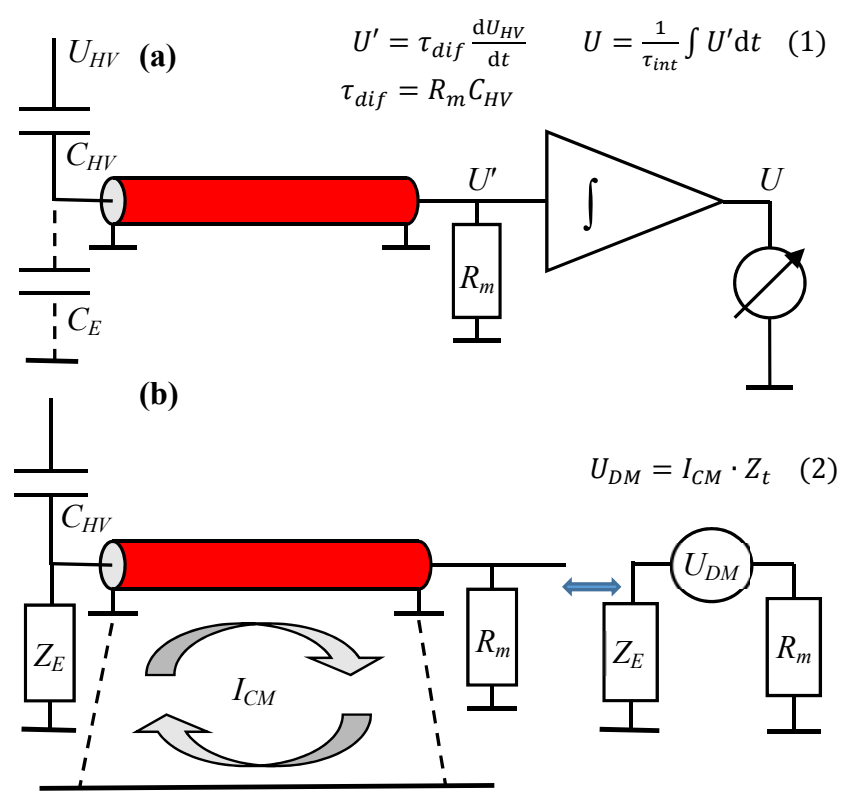

Figure 1. D/I measurement principle (a) and the effect of a common mode current (b).

In a three-phase power system, sensors can be placed nearby each phase. The relation between sensor signals and per unit phase voltages can be written as:

$$
\left(\begin{array}{l}
u_{1}\left(t ; \varphi_{0}\right) \\
u_{2}\left(t ; \varphi_{0}\right) \\
u_{3}\left(t ; \varphi_{0}\right)
\end{array}\right)=\left(\begin{array}{ccc} 
& \vdots & \\
\cdots & M_{i j} & \cdots \\
& \vdots &
\end{array}\right)\left(\begin{array}{c}
\cos (\omega t+2 / 3 \pi) \\
\cos (\omega t) \\
\cos (\omega t-2 / 3 \pi)
\end{array}\right)
$$

When fitting sinusoidal functions of a single frequency to power frequency components of a symmetric three phase system, (3) determines two parameters per row. However, matrix $\mathbf{M}$ contains nine elements and, if the time base of the recording is not synchronized with the momentary phase angle of the phase voltages, the phase shift $\varphi_{0}$ is an additional unknown [8]. The discussed applications will make use of symmetries, reducing the total number of unknowns.

\section{PD monitoring on $150 \mathrm{kV}$ terminations}

In 1994 several cable terminations simultaneously failed in the Dutch $150 \mathrm{kV}$ grid leading to an outage of several days in the affected region. It was decided to start monitoring similar type of terminations on PD activity. To obtain the PD relation with the voltage phase angle, the phase angle information needed to be extracted from the electric fields emerging from the overhead lines connected to the terminations. These terminations are in a linear configuration, see Figure 2, with equal distances between them. A sensor can be placed near the central phase, such that its couplings to the outer phases are equal. For a symmetric three-phase system the cross-couplings add to a signal in phase with the central phase. So the detected waveform would directly represent the central phase angle. The outer phases are either leading or lagging $120^{\circ}$, which can be determined by means of sensors close to terminations at the sides. However, a parallel circuit was present and its electric field would cause a phase shift in the recorded voltages. Therefore, a method employing two sensors was preferred, where the sensors were positioned such, that they were to some extent shielded from the parallel circuit while keeping a reflection symmetric configuration, see Figure 2.
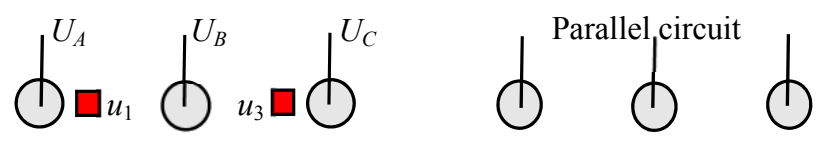

Figure 2. Positioning of two sensors (squares) nearby cable terminations; positioning at the "inside" of the termination is preferred in order to take advantage of shielding by the terminations with respect to the electric field from the parallel circuit.

\subsection{Reconstruction phase voltages}

When coefficients $\alpha, \beta, \gamma$ are assigned to the coupling strengths, ordered from a phase nearest to the sensor down to the phase furthest away, the relation between measured phasors and phasors for $U_{A}, U_{B}$ and $U_{C}$ is:

$$
\left(\begin{array}{c}
\bar{u}_{1} \\
\bar{u}_{3}
\end{array}\right)=\left(\begin{array}{ccc}
\alpha+\Delta \alpha & \beta+\Delta \beta & \gamma+\Delta \gamma \\
\gamma-\Delta \gamma & \beta-\Delta \beta & \alpha-\Delta \alpha
\end{array}\right)\left(\begin{array}{l}
\bar{U}_{A} \\
\bar{U}_{B} \\
\bar{U}_{C}
\end{array}\right)
$$

Deviations due to misalignment from perfect symmetry are included with $\Delta$ 's. As $U_{A}+U_{B}+U_{C}=0$ it follows that:

$$
\begin{aligned}
\bar{u}_{1}+\bar{u}_{3}= & -(\alpha-2 \beta+\gamma) \bar{U}_{B}+\sqrt{3}(\Delta \alpha-\Delta \gamma) \bar{U}_{B}^{\perp} \\
& \text { with } \bar{U}_{B}^{\perp} \equiv \frac{1}{3} \sqrt{3}\left(\bar{U}_{A}-\bar{U}_{C}\right)
\end{aligned}
$$

As the far end coupling and consequently its uncertainty is small compared to the main coupling, $\Delta \alpha$ governs the error in the reconstruction of the central phase: 


$$
\tan \varphi_{\text {error }}=-\frac{\sqrt{3}(\Delta \alpha-\Delta \gamma)}{\alpha-2 \beta+\gamma}
$$

This error can be reduced by, prior to adding the measured signals, normalizing them [10]. Another problem is that with the chosen sensor positioning, the denominator in (6) potentially can be close to zero because $\beta$ is not necessarily much smaller than $\alpha$. Therefore, the reconstruction can better be based on the difference of the normalized sensor signals as will be discussed in Section 3.2. The other phase references are obtained by adding or subtracting $120^{\circ}$. The phase order depends on whether the sum of the (normalized) sensor signals lags or leads their difference.

\subsection{Implementation}

To recover the phase angles, an analogue electronic device has been constructed to perform the signal manipulations. A value of $40 \mathrm{k} \Omega$ was taken for $R_{m}$ to obtain, with an estimated value for $C_{H V}$ of about $0.5 \mathrm{pF}$, a signal level close to $1 \mathrm{~V}$. Signal integration, addition and subtraction can easily be implemented with cheap standard electronic components and no heavy signal amplification is needed. It should be noted, that opting for this relatively high $R_{m}$ value does not necessarily improve the signal to noise ratio. According to Figure $1 \mathrm{~b}$ also a larger fraction from interference by ground currents could arise at the receiving end of the measurement cable.

Based on the measured and integrated signals the phasors for $u_{1}$ and $u_{3}$ are obtained. They are processed with (7) to obtain the normalized sum $U_{+}$and difference $U_{\text {- phasors: }}$

$$
\left(\begin{array}{c}
\bar{U}_{+} \\
\bar{U}_{-}
\end{array}\right)=\left(\begin{array}{c}
\text { normalize } \\
\text { resulting } \\
\text { signals }
\end{array}\right)\left(\begin{array}{c}
\text { add and } \\
\text { subtract } \\
\text { signals }
\end{array}\right)\left(\begin{array}{c}
\text { normalize } \\
\text { measured } \\
\text { signals }
\end{array}\right)\left(\begin{array}{c}
\bar{u}_{1} \\
\bar{u}_{3}
\end{array}\right)
$$

The resulting sum and difference phasors relate to the phasors for $U_{B}$ and $U_{B}{ }^{\perp}$ through a transformation matrix:

$$
\left(\begin{array}{c}
\bar{U}_{B} \\
\bar{U}_{B}^{\perp}
\end{array}\right)=\left(O_{i j}\right)\left(\begin{array}{l}
\bar{U}_{+} \\
\bar{U}_{-}
\end{array}\right)
$$

Both left and right hand side phasors are orthogonal with equal lengths as is apparent from their definitions in (5) and (7). Basically, this is because sum and difference of sinusoidal signals with equal amplitude are $90^{\circ}$ out of phase. This makes $\mathbf{O}$ an orthogonal transformation matrix. The rotation angle of $\mathbf{O}$ represents the mismatch between the phasors $U_{B}, U_{B}{ }^{\perp}$ and the reconstructed $U_{+}, U_{-}$. Laboratory tests described in $[10,11]$ showed that this error can be kept acceptable (typically $5^{\circ}$ ) by careful sensor positioning. Apparently, the effect of matrix $\mathbf{O}$ is limited, and omitting its effect still allows for an accurate reconstruction of the phase voltages.

With perfect symmetry $(\Delta$ 's $\rightarrow 0)$ matrix $\mathbf{O}$ reduces to:

$$
\mathbf{0}=\left(\begin{array}{cc}
-\operatorname{sgn}(\alpha-2 \beta+\gamma) & 0 \\
0 & \operatorname{sgn}(\alpha-\gamma)
\end{array}\right)
$$

This expression points out that there is a potential ambiguity as the sign of the first diagonal element may change. Assuming $\gamma$ is always small, this happens when $\beta$ is near $1 / 2 \alpha$. In the implementation, the reconstruction is based on $U_{B}{ }^{\perp}$ which is subsequently rotated over $90^{\circ}$. The sum signal decides which direction to rotate and also the phase order of the two other phases. The coupling of a sensor to the phase where it is installed should be sufficiently large to make sure that the sum signal is in anti-phase with phase $U_{B}$, see Section 3.3.
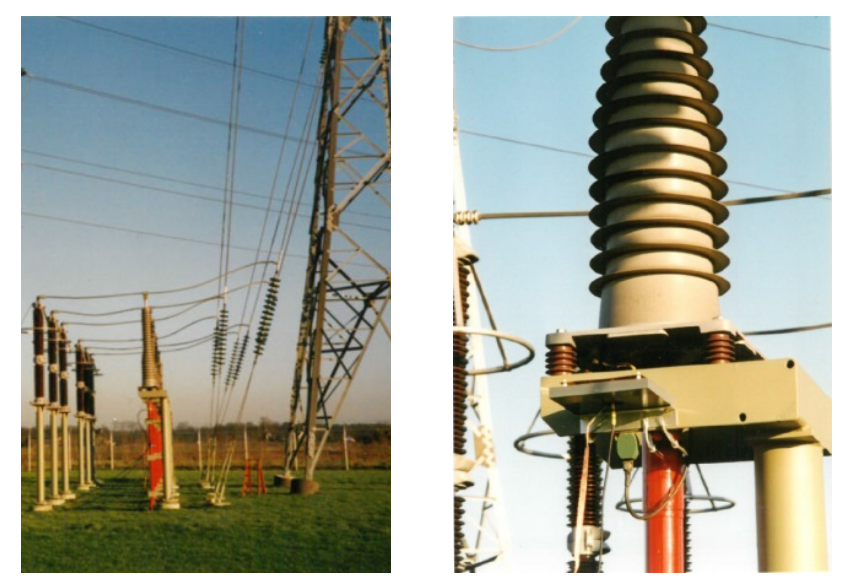

Figure 3. $150 \mathrm{kV}$ substation with two parallel circuits (left) and a capacitive sensor installed next to a termination (right).

\subsection{Field measurement}

Trial measurements were carried out at a HV substation with the overhead lines connected through a short cable length to a $150 \mathrm{kV}$ GIS system. The left photo in Figure 3 shows the terminations of two parallel circuits with surge arresters at the left of them. The right photo depicts a capacitive sensor (area $20 \mathrm{~cm} \times 20 \mathrm{~cm}$ ), attached against the supporting structure of a cable termination.

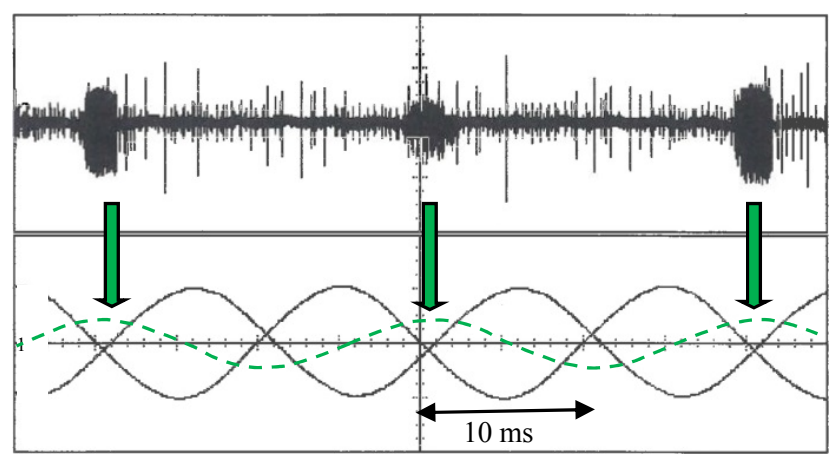

Figure 4. Recorded PD activity at phase $U_{B}$ (top) and signals from the capacitive sensors (bottom); the voltage at phase $U_{B}$, obtained from the two sensor signals, is indicated with the dashed waveform.

Figure 4 shows an actual PD measurement (top part, taken at phase $U_{B}$ ) together with the sensor signals $u_{1}$ and $u_{3}$ (bottom part). The dashed sinusoidal waveform is the reconstruction of the central phase with (7). The arrows indicate the phase voltage maxima at which highest PD activity is observed. Apparently, the PDs are corona discharges from the overhead lines rather than internal discharges within the terminations in this case. It should be noted, that the sensor signals $u_{1}$ and $u_{3}$ are close to being in anti-phase. This corresponds to (9) changing from having a determinant of -1 (rotation plus reflection) to +1 (only rotation). This would mean that the reference signal in Figure 4 could be reversed. In the realization of the equipment the signal from an additional sensor $u_{2}$ near phase $U_{B}$ can be employed instead of the sum signal to resolve this potential ambiguity. 


\section{4. $380 \mathrm{kV}$ cable to line transition point}

A $380 \mathrm{kV}$ underground cable, circuit length $10.8 \mathrm{~km}$, has been integrated in the Dutch EHV transmission grid. In 2016 and 2017 field measurements were conducted to determine overvoltages from line-energization near the transition points between power cables and overhead lines to verify whether these remain within the SIWL (switching impulse withstand level). Figure 5 depicts the topology at the transition point.
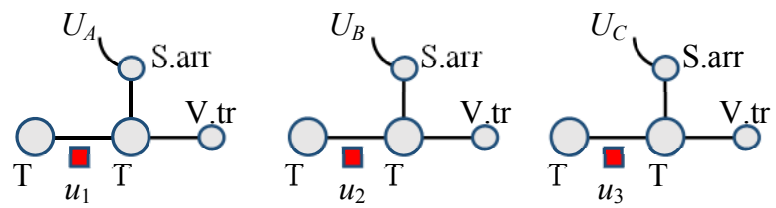

Figure 5. Schematic top view of sensor positions (squares) in between cable terminations $\mathrm{T}$ belonging to the same phase; S.arr: surge arrester, V.tr: voltage transformer.

\subsection{Coupling matrix}

Each line is connected with two power cables to match transmission capacity. Each sensor was positioned in between the terminations belonging to the same phase, providing shielding and thereby reducing cross-coupling from the other phases. Further, the topology in Figure 5 suggests that couplings from sensor 1 to phase $U_{B}$ and sensor 2 to phase $U_{C}$ are similar. Also, the couplings from sensor 2 to phase $U_{A}$ and sensor 3 to phase $U_{B}$ are similar. Deviations may occur as the lines descending from the pylons do not precisely satisfy the assumed symmetry and because of metal structures like fences in the vicinity. The matrix in (3) can be written as:

$$
\mathbf{M}=\left(\begin{array}{ccc}
M_{1} & M_{4} & f_{0} M_{1} \\
M_{5} & M_{2} & M_{4} \\
f_{0} M_{3} & M_{5} & M_{3}
\end{array}\right)
$$

Independent parameters are taken for the main couplings on the diagonal to account for deviations in symmetry. The far end couplings are low and could in principle be neglected. Electrostatic field calculations provided an estimate of about $2 \%$ of the main coupling. With aforementioned assumptions all unknowns $\left(M_{1}\right.$ to $M_{5}$ and $\left.\varphi_{0}\right)$ can be determined by fitting measured power frequency signals with an assumed perfect symmetric three-phase voltage [8]. By inverting (10) the voltages on the phase conductors upon a switching transient can be reconstructed from the measured sensor signals.

\subsection{Implementation}

Transient overvoltage measurement requires signal recording covering a wide frequency range. The measurement cable needs to be terminated with $50 \Omega$ to prevent reflections. Therefore, sufficient amplification is needed together with the signal integration to obtain signals that can be conveniently recorded by a digitizer. A hybrid passive-active integrator design published in [12] (without the proposed active offset compensation) did satisfy the requirements. In combination with the differentiating step, a straight transfer function over a frequency range from $10 \mathrm{~Hz}$ to $7 \mathrm{MHz}$ [2] is obtained with a value equal to the ratio of the differentiating and integrating time constants, see Figure 1a:
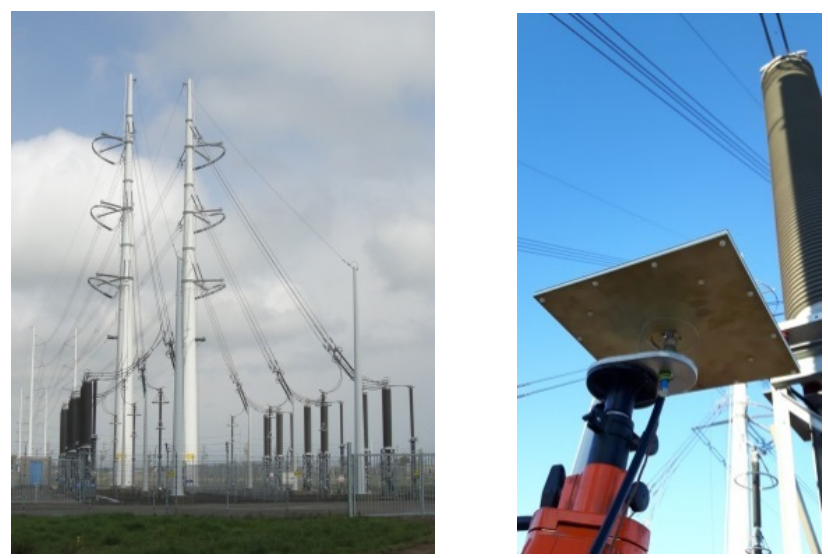

Figure 6. $380 \mathrm{kV}$ overhead line to underground power cable transition (left) and installed sensor near a cable termination (right).

$$
H_{\text {straight }}=\frac{\tau_{\text {dif }}}{\tau_{\text {int }}} \text { with } \tau_{\text {dif }}=R_{m} C_{H V}
$$

With an integrator design time constant of $20 \mu$ s and about $1 \mathrm{pF}$ coupling capacitance to the nearby phase conductor the expected signal amplitude is close to $1 \mathrm{~V}$. Sensor signals were captured with $5 \mathrm{Msample/s}$ during ten power cycles with 12 bit resolution. Figure 6 shows the configuration at one of the transition points together with an installed sensor. The sensor plate is $30 \mathrm{~cm} \times 30 \mathrm{~cm}$, placed parallel to a ground plate, separated by a $5 \mathrm{~mm}$ Teflon layer. The resulting capacitance $C_{E}$ of $300 \mathrm{pF}$ forms a low-pass filter with cut-off frequency of about $10 \mathrm{MHz}$, aiming to reduce frequencies exceeding the system's bandwidth capability from entering the active integrator.
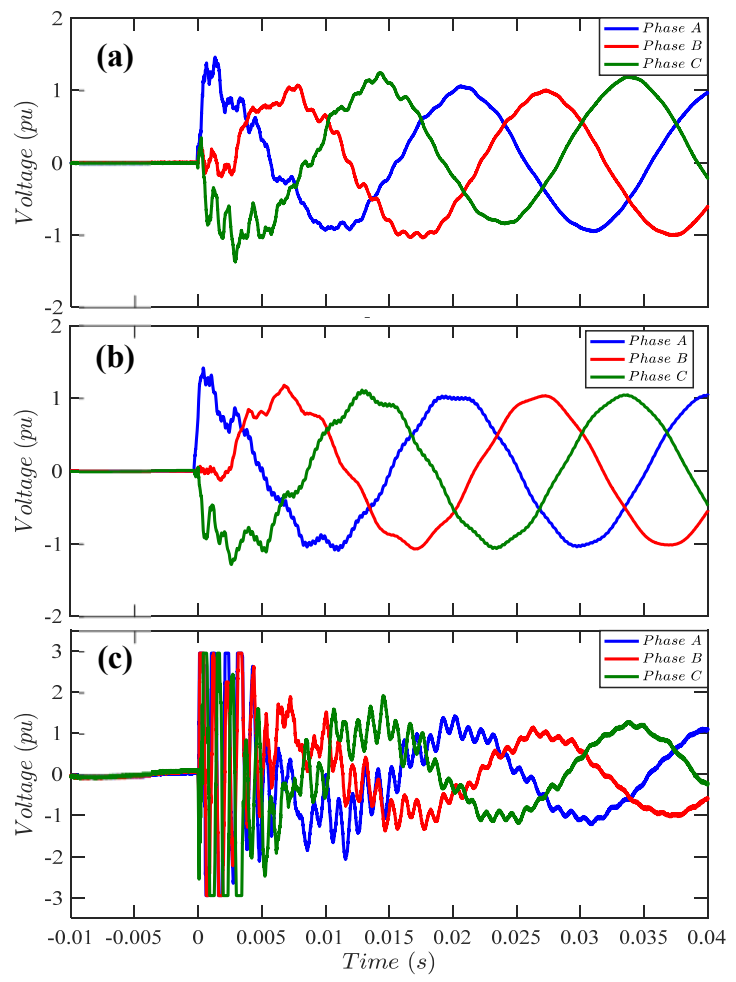

Figure 7. Energization recording with D/I system (a), PSCAD simulation (b) and $\mathrm{RC}$-divider recording (c). 


\subsection{Field Measurement}

Figure 7 provides the result from energizing at a substation $6.8 \mathrm{~km}$ away from the transition point. It shows the reconstructed D/I measurement (a), PSCAD simulation [2,7] (b) and the response of an already installed RC divider (c). The latter signal is severely disturbed, most probably because of inferior secondary cabling (high $Z_{t}$ ) in conjunction with large ground currents as discussed in Section 2. The simulated waveforms resemble the $\mathrm{D} / \mathrm{I}$ measurement result, although the oscillations in the latter are a bit stronger. This discrepancy reproduced whenever the RC-divider measurements were severely disturbed. Probably slight EMC interference may have contributed here as well. Each sensor ground plate was individually connected to the metal construction supporting the terminations. These connections together with the supporting structure make up large loops where CM-currents can be induced upon a switching event.
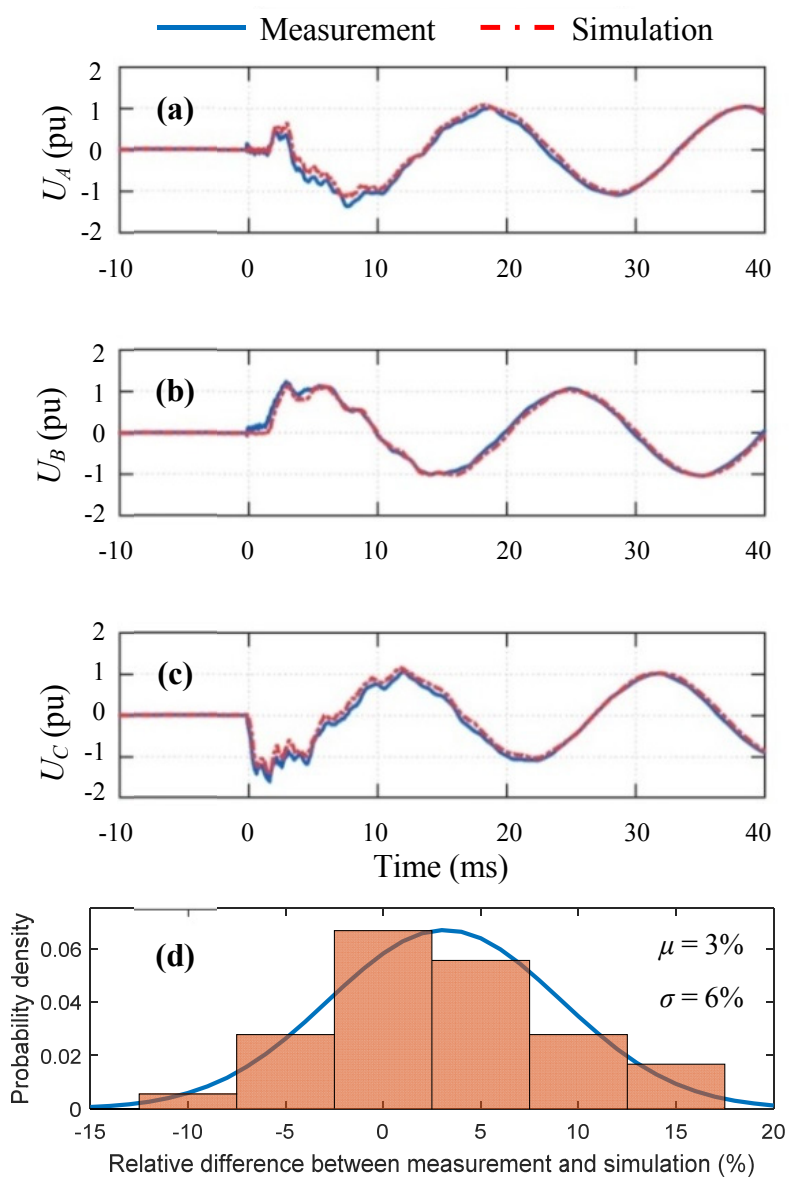

Figure 8. Comparison of measurement and simulation for two transition points upon energizing from nearby substation with parallel circuit in service for phase A-C (a-c); probability density function for percentages in deviation between measured and simulated maximum overvoltages (d).

In Figure 8a-c the results for a similar energization are shown. Here, all sensor earth wires were connected to a central earth point of the installation. This prevented common mode currents from entering the measurement cables to a large extent. The energization instances are different from Figure 7 as the energization takes place at a random moment with respect to $50 \mathrm{~Hz}$ phase angle. The differences between simulation and $\mathrm{D} / \mathrm{I}$ measurement are only minor.

Twelve energizing events are evaluated. They differ in which circuit and from which side is switched, whether the other circuit is in service and the cable termination where the measurement is taken [13]. The distribution of the relative differences between simulated and observed maximum overvoltages for the three phases is plotted in Figure 8d. It is concluded that the simulations predict the observations well. Small differences may arise as the transient overvoltages also depend on the downstream networks which were only modelled with equivalent lumped loads [7].

\section{Switching at $380 \mathrm{kV}$ substation}

The main strength of the $\mathrm{D} / \mathrm{I}$ principle is its capability to capture high frequencies [3] with good EMC characteristics. For low frequencies the (active) integration step inherently has a struggle with its offset compensation. Regarding this aspect RC-dividers are more convenient. To observe fast front overvoltages, similar switching as in Section 4 has been recorded. The equipment was installed at the substation where the circuit breaker is activated, see Figure 9.

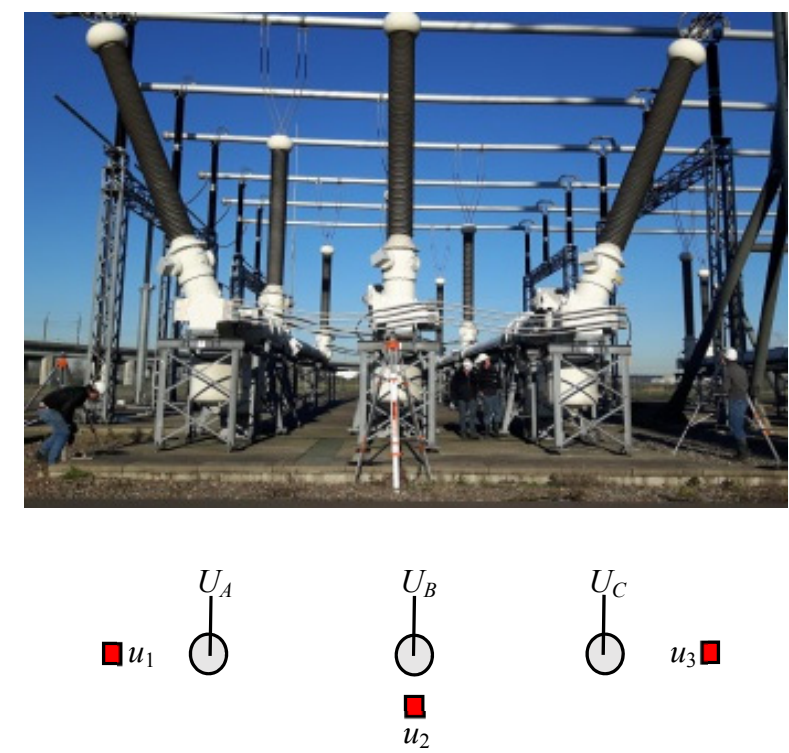

Figure 9. Substation (top) and schematic configuration of sensors, indicated as squares, with respect to the phase conductors (bottom).

\subsection{Field Measurement}

Positioning of the sensors at the substation could not be achieved without significant cross-coupling and larger effect of assumptions in the decoupling are to be expected. In [8] the propagation of such uncertainties is analysed. The result is shown in Figure 10. The black lines in Figure 10a indicate one standard deviation margins based on assumed uncertainties in the coupling matrix parameters. The zoomed figures show the travelling waves reflecting at the line to cable transition. The energization of the phase conductors occurs at different time instances. In particular, one phase is energized with a delay of 
over $0.5 \mathrm{~ms}$, when it is close to its peak value. This results in a short spike. The bottom waveform shows that the initial peak is only one sample point wide (indicated with an arrow), suggesting that the sampling rate and the analogue bandwidth, were still too low for good time resolution.
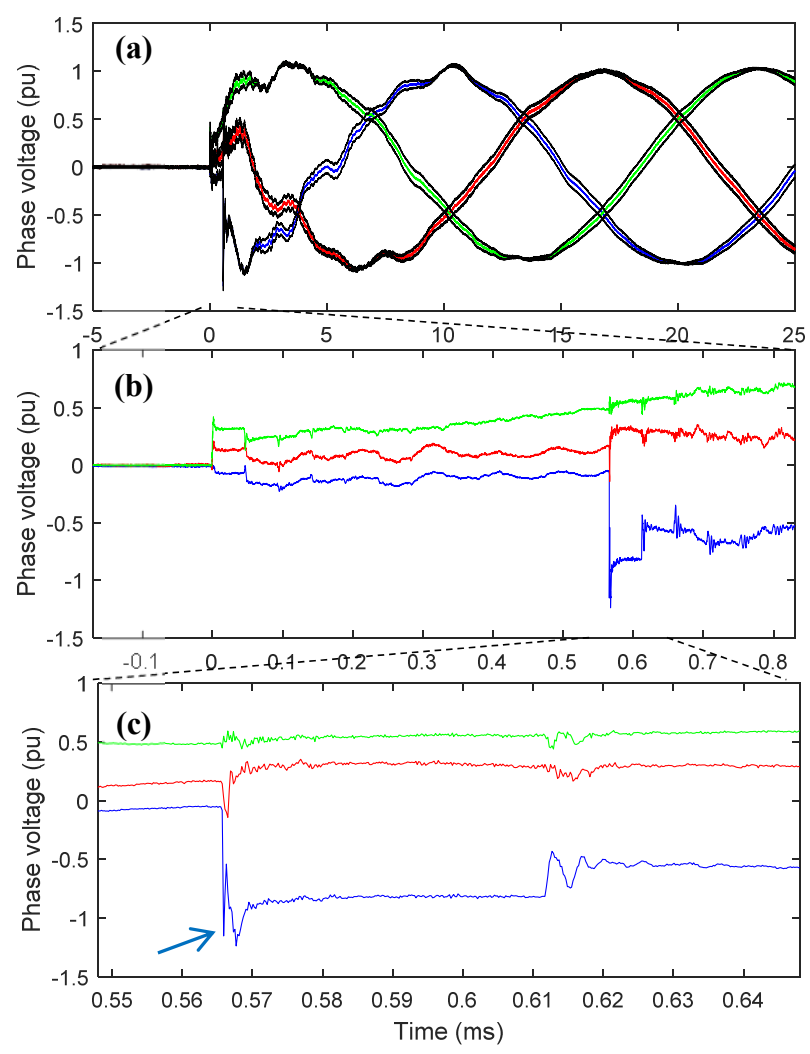

Figure 10. Transients at substation (a) with 30 times (b) and 300 times (c) zoomed waveforms near the energizing moment.

\subsection{Perspectives for VFTO measurement}

The presence of narrow spikes raises the question on the limits of the $\mathrm{D} / \mathrm{I}$ system in the sense of its capability to record very fast transient overvoltages (VFTO) with frequencies up to e.g. $100 \mathrm{MHz}$ [1]. The value of $C_{E}$ must obviously be lowered. A factor of ten can easily be achieved by increasing the distance between the sensor and ground plates and replace the Teflon layer by air insulation, see Section 4.2. The main limitation is the integrator design with operational amplifiers. Instead, one could use only a passive integrator. To achieve the same sensitivity as before, i.e. having the same integrator time constant of $20 \mu$ s (e.g. a passive integrator with $5 \mathrm{k} \Omega$ and $4 \mathrm{nF}$ ), the detection bandwidth would then start as from about $8 \mathrm{kHz}$.

The high-frequency cut-off is ultimately determined by the length of the line effectively observed by the sensor. The front time associated with $100 \mathrm{MHz}$ corresponds to $3 \mathrm{~m}$ wavelength for propagation with the speed of light. Such small observation length can only be achieved for capacitive sensors e.g. inside a compact GIS. In open systems a longer line length will contribute to the sensor signals, limiting the upper detectable frequency to at most an estimated value of $30-50 \mathrm{MHz}$.

\section{Conclusion}

With open-air capacitive sensors an easy to install measurement system was realized for field measurement of (over)voltages in $\mathrm{HV}$ and EHV grids. The cross-coupling inherent to the use of open-air sensors can be dealt with and the superior EMC characteristics as part of the $\mathrm{D} / \mathrm{I}$ principle makes it an attractive alternative for the use of $\mathrm{RC}$ dividers, in particular when no permanent installation is desired. The technique can also be applied to capture VFTOs up to several tens of megahertz.

For the near future, measurements are planned to observe switching overvoltages at compensation coils connected to the $50 \mathrm{kV}$ tertiary transformer windings in the Dutch $380 \mathrm{kV}$ grid. The combination of a circuit breaker very near to a transformer has the potential to cause very fast transient overvoltages.

\section{References}

[1] Hugh M. Ryan (ed.), High-Voltage Engineering and Testing, IET Power and Energy Series, 66, $3^{\text {rd }}$ edition, 2013.

[2] F. Barakou, P.A.A.F. Wouters, S. Mousavi Gargari, J. Smit, E.F. Steennis, "Merits and challenges of a differentiating-integrating measurement methodology with air capacitors for high-frequency transients", CIGRE, C4-203, Paris, August 2018.

[3] E.J.M. van Heesch, A.P.J. van Deursen, M.A. van Houten, G.A.P. Jacobs, W.F.J. Kersten and P.C.T. van der Laan, "Field tests and response of the D/I H.V. measuring system", $6^{\text {th }}$ International Symposium on High-Voltage Engineering, New Orleans, August 1989.

[4] E.J.M. van Heesch, R. Caspers, P.F.M. Gulickx, G.A.P. Jacobs, W.F.J. Kersten and P.C.T. van der Laan, "Three phase voltage measurements with simple open air sensors", $7^{\text {th }}$ International Symposium on High-Voltage Engineering, Dresden, August 1991.

[5] R.P.P. Smeets, W.A. van der Linden, M. Achterkamp, G.C. Damstra and E.M. de Meulemeester, "Disconnector switching in GIS: three-phase testing and phenomena", IEEE Transactions on Power Delivery, Vol. 15, No. 1, 2000, pp. 122-127.

[6] Kun-Long Chen, Yi Guo, Xiaoyu Ma, "Contactless voltage sensor for overhead transmission lines", IET Generation, Transmission \& Distribution, Vol. 12, No. 4, 2018, pp. 957-966.

[7] F. Barakou, P.A.A.F. Wouters, S. Mousavi Gargari, J.P.W. de Jong, E.F. Steennis, "Online transient measurements of EHV cable system and model validation", IEEE Transactions on Power Delivery, Vol. 34, No. 2, 2019, pp. 532-541.

[8] P.A.A.F. Wouters, F. Barakou, S. Mousavi Gargari, J. Smit and E.F. Steennis, "Accuracy of switching transients measurement with open-air capacitive sensors near overhead lines", IEEE International Conference on High Voltage Engineering and Application, Athens, September 2018.

[9] P.C.T. van der Laan and A.P.J. van Deursen, "Reliable protection of electronics against lightning: some practical applications", IEEE Transactions on Electromagnetic Compatibility, Vol. 40, No. 4, 1998, pp. 513-520.

[10]P. Wouters, S. van den Broek, E. van Heesch, P. van der Laan, "Capacitieve bepaling fasehoeken 3-fasen hoogspanningssysteem", internal report EHC/RAP/94048 in Dutch, Technische Universiteit Eindhoven, November 1994.

[11] P.A.A.F. Wouters, M.J.A.M. van Helvoort, P.R. Bruins, "Fasehoekdetectie aan een $50 / 60 \mathrm{~Hz}$ 3-fasen hoogspanningssysteem", internal report EHC/RAP/95059 in Dutch, Technische Universiteit Eindhoven, September 1995.

[12] A.P.J. van Deursen, H.W.M. Smulders, R.A.A. de Graaff, "Differentiating/integrating measurement setup applied to railway environment", IEEE Transactions on Instrumentation and Measurement, Vol 55, No. 1, 2006, pp. 316-326.

[13]F. Barakou, "Investigation of the impact of EHV underground power cables on the resonant and transient grid behavior", Dissertation Eindhoven University of Technology, December 2018. 